\title{
BIQQ-BYWORD
}

\section{Water Quality Microbiological Inspection and Quality Control Technology}

\author{
Ran Chi' ${ }^{*}$, Chen Sun' ${ }^{1}$, Siyi Li ${ }^{2}$ \\ ${ }^{1}$ Shanghai Shuiyuandi Constructi \& Development Co., Ltd., Shanghai 200000, China \\ ${ }^{2}$ Liaoning Fengtian Testing Technology Co., Ltd., Shenyang 100000, Liaoning Province, China \\ *Corresponding author: Ran Chi, ran.chi@poten.cn
}

\begin{abstract}
People's lives are strongly linked to the quality of their drinking water. The quality of drinking water can be successfully ensured by testing microorganisms in water quality samples and examining their unqualified variables. Many urban residents' drinking water contains a variety of microorganisms, which will have a direct impact on the quality of the water, and all microorganisms will offer a health risk. As a result, steps should be taken to eliminate germs. As a result, the focus of this article is on water quality microbiological inspection and quality control technology.
\end{abstract}

Keywords: Water microorganism; Inspection; Quality control

Publication date: November 2021; Online publication: November 30, 2021

\section{Introduction}

The inspection of water quality microorganisms not only plays a great role in promoting the safe use of drinking water, but also plays a very important role in ecological balance. Therefore, the corresponding inspection staff should well control the inspection quality and adopt effective quality control methods to ensure the comprehensive development of the inspection work, so as to lay a solid foundation for the inspection and safe use of drinking water in our country.

\section{Microbiological examination and analysis of water quality}

\subsection{Sample collection}

From April 2018 to February 2019, according to the population proportion of a city, the monitoring of drinking water quality was completed at 168 monitoring points in the city, mainly including tap water monitoring points in the city, small centralized water supply points and decentralized water supply points in the city. A total of 4400 drinking water samples were collected (of which 2200 were analyzed for urban and rural drinking water quality in different seasons; The other 2200 samples were used to analyze the difference between the total number of bacteria, total coliform group and heat-resistant coliform group in urban and rural water). After drinking water samples were sampled, they were directly sent to the laboratory within 4.5 hours, and then the samples were tested in a scientific way.

\subsection{Test method}

The detection of drinking water is a complex work. During the specific detection period, the main content is to test the microorganisms in drinking water. The main inspection contents include the total number of colonies, coliform group, heat-resistant coliform group and so on. When detecting the heat-resistant coliform bacteria and total coliform liver bacteria in drinking water samples, the multi tube fermentation method is generally used to complete the corresponding detection. When detecting the total number of 
colonies, the plate counting method is usually used to ensure the rationality and accuracy of the final detection results.

\subsection{Results}

\subsubsection{Sample qualification}

Among the 2200 drinking water standards adopted, there are 2000 qualified samples. After analysis, there is a significant difference in the qualified rate of drinking water samples between rural and urban areas, and there is a significant difference in the qualified rate of drinking water between summer, autumn and spring and winter $(\mathrm{P}<0.05)$. The specific situation is shown in Table 1.

Table 1. Sample testing

\begin{tabular}{ccccc}
\hline \multirow{2}{*}{ Program } & \multicolumn{2}{c}{ Season } & \multicolumn{2}{c}{ Region } \\
\cline { 2 - 5 } & Summer and Autumn & Spring and Winter & Village & City \\
\hline Number of samples (copies) & 1325 & 875 & 1280 & 920 \\
Number of qualified & 1225 & 775 & 1190 & 810 \\
samples (copies) & 0.000 & & 0.000 & \\
P & & & & \\
\hline
\end{tabular}

Table 2. Inspection of microbiological indexes of residents' drinking water

\begin{tabular}{ccccc}
\hline \multirow{2}{*}{ Group } & \multicolumn{2}{c}{ City } & \multicolumn{2}{c}{ Village } \\
\cline { 2 - 5 } & $\begin{array}{c}\text { Number of } \\
\text { samples (copies) }\end{array}$ & $\begin{array}{c}\text { Number of qualified } \\
\text { samples (copies) }\end{array}$ & $\begin{array}{c}\text { Number of } \\
\text { samples (copies) }\end{array}$ & $\begin{array}{c}\text { Number of qualified } \\
\text { samples (copies) }\end{array}$ \\
\hline $\begin{array}{c}\text { Aerobic bacterial } \\
\text { count }\end{array}$ & 400 & 390 & 400 & 328 \\
$\begin{array}{c}\text { Total coliform } \\
\text { Thermotolerant }\end{array}$ & 400 & 384 & 400 & 331 \\
coliform bacteria & 300 & 286 & 300 & 242 \\
\hline
\end{tabular}

In the 2200 drinking water standards adopted, the inspection statistics of various microbial indicators of residents' drinking water are shown in Table 2. As can be seen from the median in Table 2, the rational rate of drinking water in urban areas is higher than that in rural areas, no matter the total number of bacteria, total coliform and heat-resistant coliform. The test results show that the quality of drinking water in cities is significantly better than that in rural areas. One of the main reasons for this phenomenon is that the water sources in cities and rural areas are different, and the treatment of drinking water is also different.

\subsubsection{Microbiological examination}

In the microbiological examination of 2200 water samples, the total number of colonies was 2052 , the total coliform group was 1998, and the heat-resistant coliform group was 2008.

\subsection{Microbiological examination findings}

In the process of drinking water inspection, microbial inspection method is one of the commonly used methods. It can quickly and effectively detect the microbial species and content contained in drinking water. It is an important inspection method to ensure the safety of drinking water and escort people's health. In this test, the collected water samples are divided into two groups, and two microbial test methods are used 
respectively. These two test methods are more commonly used and can get good test results. Among them, the test effect of filter membrane test method is better, but the detection rate is relatively low compared with multi tube fermentation test method. On the contrary, after using MPN test, the detection rate of multi tube fermentation test method is improved on the basis of ensuring the accuracy of test results. It can be seen that in the process of drinking water inspection, the inspection effect and detection rate of multi tube fermentation method are higher than that of filter membrane inspection method, which can better improve drinking water quality, ensure drinking water safety and build a good drinking water environment for people.

\section{Quality control technology for microbiological inspection of water quality}

After understanding the common factors affecting the microbiological test results of water quality, we will start from the detailed aspects to explore the scheme to reduce the adverse effects. According to different influencing factors, specific solutions are put forward to control the progress of testing work and the quality of testing results. On the premise of ensuring the smooth development of testing work, optimize the testing process, create a good environment for testing work and fundamentally promote the improvement of testing level.

\subsection{Level of testing personnel}

The relevant staff engaged in water quality microbiological inspection must have rich accumulation reserves of professional basic knowledge of microbiological inspection, understand and be familiar with the relevant operation process of water quality microbiological inspection, and avoid the inaccurate test results in water quality microbiological inspection caused by their own professional quality and professional skill level is not up to standard ${ }^{[1]}$. Therefore, relevant departments must put forward strict requirements for relevant inspectors of water quality microbiological testing. Before carrying out water quality microbiological testing, relevant knowledge training and comprehensive assessment shall be organized to ensure that inspectors have the basic conditions for experimental development and master the operation process of water quality microbiological testing.

Relevant staff involved in microbiological testing must receive systematic professional training before taking up their posts. Inspectors need to firmly grasp the testing operation skills, relevant theoretical knowledge, testing standard requirements and practical operation methods, and pass strict examination before taking up their posts. Moreover, in the process of monitoring, we must work in strict accordance with relevant documents or instructions, and regularly carry out professional training for testers. We should not only improve the professional skills of testers, but also pay attention to the professional quality of testers, so as to ensure that effective measures can be taken to control the accuracy of testing in practical work. The testing laboratory should establish and improve the responsibility system, truly implement the responsibilities of each testing personnel, and avoid shirking responsibilities after problems in the testing results, so as to obtain more accurate testing results and improve the efficiency and quality of testing work.

\subsection{Control of instruments and equipment's}

Instruments and equipment are essential hardware support for microbial detection, which can directly affect the detection results. Therefore, in the process of microbial testing, it is necessary to accurately manage relevant instruments and equipment, carry out actual operation according to the rules and regulations formulated by relevant departments, regularly inspect and maintain all equipment, and cultivate the safety awareness of all testing personnel. Not only that, the verification work must also be done in place to ensure that all equipment and instruments have reached the standard that can start detection, so as to realize standardized microbial detection. In microbiological testing, microscope is one of the important equipment. It can carefully observe the morphology of microorganisms in water. After using the microscope, the optical 
glass and stage should be thoroughly cleaned with lens wiping paper to avoid contamination of the next observed sample. At the same time, attention should be paid to the anti-corrosion, anti-vibration, anti-dust and anti-moisture maintenance of the microscope.

\subsection{Control of environment factors}

During microbial testing, the surrounding environment will have a direct impact on the test results. Therefore, when carrying out the actual monitoring work, the relevant inspectors must establish a correct work awareness and work concept, and follow the scientific and reasonable working principles to ensure that the control effect of the test results is improved. Installing ventilation equipment in the laboratory can avoid the rapid change of large air flow or too many floating and sinking objects in the air affecting the test results. Installing air conditioning in the laboratory can not only prevent the pollution of water samples by bacteria, but also ensure the stable operation of incubator equipment and reduce the moisture problem of instruments, equipment and culture medium ${ }^{[2]}$. We need to prevent the pollution of water samples and avoid the pollution of water samples by the surrounding environment, or the mutual pollution between different water samples. Therefore, the layout of the laboratory should be reasonable, and different distinguishing signs should be set in different functional areas to avoid affecting the test. In addition, the air inside the laboratory should also be monitored in real time. Only after it determining that the air has reached the detection standard, the microbial detection can be formally carried out.

\subsection{Effectively maintain samples to slow down the propagation of microorganisms}

Generally, samples are kept at low temperature. After all samples are collected, they will be sent to the laboratory immediately. In the process of sample transportation, pay attention not to break the glass bottle, minimize the contact between foreign objects and the bottle body, and fix the glass bottle to avoid sample loss and dumping.

\subsection{Use sterile glass bottle, and strictly require the consistency of sample collection}

With the passage of time and space, the growth and classification of microorganisms will change greatly. Therefore, in the process of detecting microorganisms in water, the collected samples must be representative to a certain extent, and the consistency of samples must be ensured. For example, samples are collected in the same water area and at the same time, and they are collected directly. Sterile glass bottles are not allowed to be used for collection, so as to avoid the pollution of other bacteria and affect the detection and treatment.

\subsection{Quality control of microbial sample collection for water quality monitoring}

Many types of water will alter dramatically over time and space, necessitating the development of a sensible sample strategy. The water intake point must be fixed at the same time to ensure that the obtained water samples are representative. To thoroughly mix during inspection, the water extraction volume must be 80 percent of the bottle capacity; when sampling, the sterilized sampling bottle must be collected directly, not brushed with water sample, and contamination of the bottle mouth by fingers and other objects must be avoided. During sampling, do not stir the sediment at the bottom of the water. Before taking water samples from the pipe network, sterilize the faucet. The procedure is to use a flame to burn the faucet surface for a few seconds (3-5 seconds), then drain the water for 5-10 minutes. Reservoirs are found in some rural residential water samples. Prepare a clean bucket and place it in the pool for few minutes.

\section{Conclusion}

In the water quality monitoring work, there have been some problems in the inspection of microorganisms 
in water, such as the standard analysis of microorganisms and how to find effective reference samples. These situations must be analyzed in detail. Therefore, there are certain requirements for the working experience of staff, and personal experience judgment is more important in the inspection work. The inspection process of water quality microorganisms must be from sample collection to analysis of inspection results. These inspection results have a certain impact on sample collection technology, inspectors and experimental steps.

\section{Disclosure statement}

The author declares no conflict of interest.

\section{References}

[1] Li HM, 2020, Microbiological Test and Quality Control Measures of Water Quality. Clinical Laboratory Journal (Electronic Edition), 9(01): 235.

[2] Wu YC, 2019, Causes and Countermeasures of Unqualified Microbiological Samples in Water Quality. Journal of Inspection and Quarantine, 29(06): 112-113. 